\title{
Integrating Text Plans for Conciseness and Coherence*
}

\author{
Terrence Harvey and Sandra Carberry \\ Department of Computer Science \\ University of Delaware \\ Newark, DE 19716 \\ \{harvey,carberry\}@cis.udel.edu
}

\begin{abstract}
Our experience with a critiquing system shows that when the system detects problems with the user's performance, multiple critiques are often produced. Analysis of a corpus of actual critiques revealed that even though each individual critique is concise and coherent, the set of critiques as a whole may exhibit several problems that detract from conciseness and coherence, and consequently assimilation. Thus a text planner was needed that could integrate the text plans for individual communicative goals to produce an overall text plan representing a concise, coherent message.

This paper presents our general rule-based system for accomplishing this task. The system takes as input a set of individual text plans represented as RST-style trees, and produces a smaller set of more complex trees representing integrated messages that still achieve the multiple communicative goals of the individual text plans. Domain-independent rules are used to capture strategies across domains, while the facility for addition of domain-dependent rules enables the system to be tuned to the requirements of a particular domain. The system has been tested on a corpus of critiques in the domain of trauma care.
\end{abstract}

\section{Overview}

Many natural language systems have been developed to generate coherent text plans (Moore and Paris, 1993; Hovy, 1991; Wanner and Hovy, 1996; Zukerman and McConachy, 1995). However, none has the ability to take a set of independently generated yet inter-related text plans and produce integrated plans that realize all of the communicative goals in a concise and coherent manner.

RTPI (Rule-based Text Plan Integrator) was designed to perform this task. The need for coherence requires that the system be able to

\footnotetext{
* This work was supported by the National Library of Medicine under grant R01-LM-05764-01. We thank Bonnie Webber and John Clarke for their suggestions and advice during the course of this research.
}

identify and resolve conflict across multiple, independent text plans, and exploit relations between communicative goals. Conciseness requires the ability to aggregate and subsume communicative goals. Although our work was motivated by the need to produce coherent, integrated messages from the individual critiques produced by a decision support system for emergency center trauma care, this same task will arise in future systems as they make use of independent modules that need to communicate with a user. Thus the system should have simple, domain-independent rules, but should also be flexible enough to allow the addition of rules specific to the domain at hand.

This paper describes $R T P I$ and our initial implementation that works with the kinds of text plans representative of a critiquing system. While our examples are taken from the domain of trauma care, the domain-independent rules make the system applicable to other domains of critiquing and instruction as well. The motivation behind $R T P I$ is presented in Section 2, and Section 3 contrasts it with other work. Then we describe the system's parameters that allow flexible response in multiple environments (Section 4). The heart of the system is RTPTs domain-independent rule base (Section 5 ) for integrating text plans. The implemented algorithm and the results of its application are presented last.

\section{Motivation}

TraumAID (Webber et al., 1992) is a decision support system for addressing the initial definitive management of multiple trauma. TraumaTIQ (Gertner and Webber, 1996) is a module that infers a physician's plan for managing patient care, compares it to TraumAID's plan, and critiques significant differences between them. TraumaTIQ recognizes four classes of differences: errors of omission, errors of commission, scheduling errors, and procedure choice errors. Experimentation with TraumaTIQ showed that when the physician's plan is deficient, several problems are generally detected, and thus multiple critiques are independently produced. 
We analyzed 5361 individual critiques comprising 753 critique sets produced by TraumaTIQ on actual cases of trauma care. A critique set represents the critiques that are produced at a particular point in a case. While each critique was coherent and concise in isolation, we found several problems within critique sets: some critiques detracted from others in the critique set; some would make more sense if they took explicit account of other critiques appearing earlier in the set; and there was informational overlap among critiques.

Our analysis revealed 22 common patterns of inter-related critiques, each pattern covering some subset of a critique set. While we initially developed a domain-dependent system, TraumaGEN, that operated directly on the logical form of the critiques produced by TraumaTIQ, we noted that many of the patterns were more generally applicable, and that the problems we were addressing would also arise in other sophisticated systems that distribute their processing across multiple independent modules, each of which may need to communicate with the user. While such systems could be designed to try to prevent problems of this kind from arising, the result would be less modular, more complex, and more difficult to extend.

Thus we developed RTPI, a system for constructing a set of integrated RST-style text plans from a set of individual text plans. RTPI contains a set of domain-independent rules, along with adjustable parameters that determine when and how rules are invoked. In addition, RTPI allows the addition of domaindependent rules, so the system can account for interactions and strategies particular to a domain.

\section{Other Work}

The idea of domain-independent text planning rules is not new. Appelt (1985) used "interactions typical of linguistic actions" to design critics for action subsumption in KAMP. REVISOR (Callaway and Lester, 1997) used domainindependent operators for revision of a text plan for explanation. Because our rules operate on full RST-style text plans that include communicative goals, the rules can be designed to integrate the text plans in ways that still satisfy those goals.

The Sentence Planner (Wanner and Hovy, 1996) uses rules to refine a single initial tree representation. In contrast, $R T P I$ operates on sets of complete, independent text plan trees. And while REVISOR handles clause aggregation, and Sentence Planner removes redundancies by aggregating neighboring expressions, neither of them addresses the aggregation of communicative goals (often requiring reorganization), the
TraumaTIQ critiques:

Caution: check for medication allergies, and do a laparotomy immediately to treat the intra-abdominal injury.

Consider checking for medication allergies now to treat a possible GI tract injury.

Please remember to check for medication allergies before you give antibiotics.

Message from RTPI integrated plan:

Caution: check for medication allergies to treat the intra-abdominal injury and a possible GI tract injury, and do it before giving antibiotics. Then do a laparotomy to complete treating the intra-abdominal injury.

Figure 1: Result of communicative goal aggregation.

revision and integration of text plans to remove conflict, or the exploiting of relations between communicative goals as done by RTPI. Similarly, WISHFUL (Zukerman and McConachy, 1995) includes an optimization phase during which it chooses the optimal way to achieve a set of related communicative goals. However, the system can choose to eliminate propositions and does not have to deal with potential conflict within the information to be conveyed.

\section{System Parameters}

Although RTPTs rules are intended to be domain-independent, environmental factors such as the purpose of the messages and the social role of the system affect how individual text plans should be integrated. For example, if the system's purpose is to provide directions for performing a task, then an ordered set of actions will be acceptable; in contrast, if the system's purpose is decision support, with the user retaining responsibility for the selected actions, then a better organization will be one in which actions are grouped in terms of the objectives they achieve (see Section 5.1.1). Similarly, in some environments it might be reasonable to resolve conflict by omitting communicative goals that conflict with the system's action recommendations, while in other environments such omission is undesirable (see Section 5.1.2).

$R T P I$ has a set of system parameters that capture these environmental factors. These parameters affect what rules are applied, and in some cases how they are applied. They allow characteristics of the output text plans to be tailored to broad classes of domains, giving the system the flexibility to be effective over a wide range of problems. 


\section{The Rule-Base}

$R T P \Gamma \mathrm{s}$ input consists of a set of text plans, each of which has a top-level communicative goal. Rhetorical Structure Theory (Mann and Thompson, 1987) posits that a coherent text plan consists of segments related to one another by rhetorical relations such as MOTIVATION or BACKGROUND. Each text plan presented to RTPI is a tree structure in which individual nodes are related by RST-style relations. The top-level communicative goal for each text plan is expressed as an intended effect on the user's mental state (Moore, 1995), such as (GOAL USER (DO ACTION27)). The kinds of goals that $R T P I$ handles are typical of critiquing systems, systems that provide instructions for performing a task, etc. These goals may consist of getting the user to perform actions, refrain from performing actions, use an alternate method to achieve a goal, or recognize the temporal constraints on actions.

Rules are defined in terms of tree specifications and operators, and are stylistically similar to the kinds of rules proposed in (Wanner and Hovy, 1996). When all the tree specifications are matched, the score function of the rule is evaluated. The score function is a heuristic specific to each rule, and is used to determine which rule instantiation has the best potential text realization. Scores for aggregation rules, for example, measure the opportunity to reduce repetition through aggregation, subsumption, or pronominal reference, and penalize for paragraph complexity.

Once a rule instantiation is chosen, the system performs any substitutions, pruning, and moving of branches specified by the rule's operators. The rules currently in use operate on text plan trees in a pairwise fashion, and recursively add more text plans to larger, already integrated plans.

\subsection{Classes of Rules}

$R T P I$ has three classes of rules, all of which produce an integrated text plan from separate text plans. The classes of rules correlate with the three categories of problems that we identified from our analysis of TraumaTIQ's critiques, namely, the need to: 1) aggregate communicative goals to achieve more succinct text plans; 2 ) resolve conflict among text plans; and 3 ) exploit the relationships between communicative goals to enhance coherence.

\subsubsection{Aggregation}

Our analysis of TraumaTIQ's output showed that one prevalent problem was informational overlap, i.e. the same actions and objectives often appeared as part of several different input text plans, and thus the resulting messages

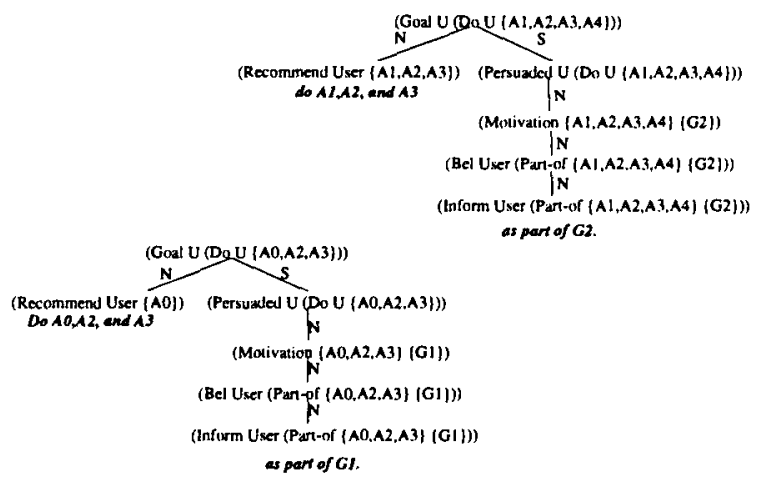

Figure 2: Input to RTPI (see Figure 3).

appear repetitious. Aggregation of the communicative goals associated with these actions and objectives allows RTPI to make the message more concise.

Aggregation of overlapping communicative goals is not usually straightforward, however, and often requires substantial reorganizing of the trees. Our approach was to draw on the ordered, multi-nuclear SEQUENCE relation of RST. We posited that separate plans with overlapping communicative goals could often be reorganized as a sequence of communicative goals in a single plan. The recommended actions can be distributed over the sequentially related goals as long as the new plan captures the relationships between the actions and their motivations given in the original plans.

For example, one complex class of aggregation is the integration of text plans that have overlapping actions or objectives, but also contain actions and objectives that do not overlap. When those that overlap can be placed together as part of a valid sequence, a multi-part message can be generated. $R T P I$ produces an integrated text plan comprised of sequentially related segments, with the middle segment conveying the shared actions and their collected motivations. The other segments convey the actions that temporally precede or follow the shared actions, and are also presented with their motivations. For example (Fig. 5), suppose that one text plan has the goal of getting the user to perform actions $A 0, A 2$, and $A 3$ to achieve $G 1$, while a second text plan has a goal of getting the user to perform $A 1, A 2, A 3$, and $A 4$ to achieve $G 2$. Figure 3 presents the text plan resulting from the application of this rule. Realization of this text plan in English produces the message:

Do $A 0$ as part of $G 1$, and $A 1$ as part of $G 2$. Next do $A 2$ and $A 3$ to address both of these goals. Then do A4 to complete G2. 


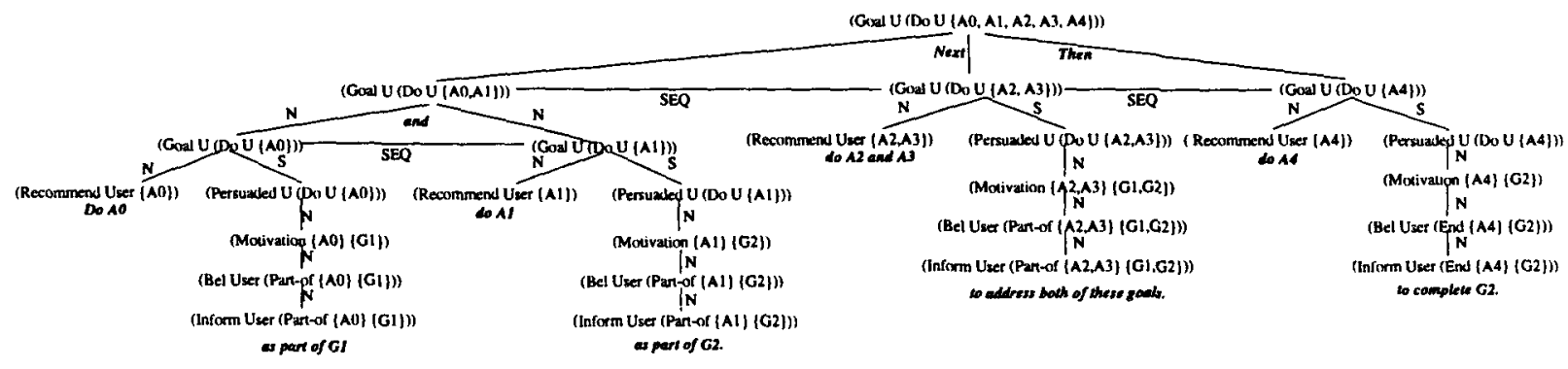

Figure 3: Result of a complex aggregation rule (see Figure 2).

This kind of aggregation is especially appropriate in a domain (such as trauma care) where the clause re-ordering normally applied to enable aggregation (e.g. Sentence Planner) is restricted by the partial ordering of sequenced instructions.

$R T P I$ can also handle aggregation when actions or objectives are shared between different kinds of communicative goals. The bottom part of Figure 1 is the text realized from a text plan that was produced by the application of two rules to three initial text plans: one rule that applies to trees of the same form, and one that applies to two distinct forms. The first rule aggregates the communicative goal (GOAL USER (DO USER check_med_allergies)) that exists in two of the text plans. The second rule looks for overlap between the communicative goal of getting the user to do an action and the goal of having the user recognize a temporal constraint on actions. The application of these two rules to the text plans of the three initial messages shown in the top part of Figure 1 creates the integrated text plan shown in Figure 4 whose English realization appears in the bottom part of Figure 1.

RTPT's parameter settings capture aspects of the environment in which the messages will be generated that will affect the kind of aggregation that is most appropriate. The settings for aggregation determine whether RTPI emphasizes actions or objectives. In the latter case (appropriate in the trauma decision-support environment), an arbitrary limit of three is placed on the number of sequentially related segments in a multi-part message, though each segment can still address multiple goals. This allows the reorganization of communicative goals to enable aggregation while maintaining focus on objectives.

\subsubsection{Resolving Conflict}

The ability to recognize and resolve conflict is required in a text planner because both the appearance and resolution of conflict can be the result of text structure. $R T P I$ identifies and resolves a class of domain-independent conflict, with the resolution strategies dependent upon the social relationship between the user and the system. In addition, the system allows the user to add rules for domain-specific classes of conflict.

One class of conflict that can best be resolved at the text planning level results from implicit messages in text. Resolving conflict of this kind within independent modules of a critiquing system would require sharing extensive knowledge, thereby violating modularity concepts and making the planning process much more complex.

For example, suppose that the user has conveyed an intention to achieve a particular objective by performing act $A_{u}$. One system module might post the communicative goal of getting the user to recognize that act $A_{p}$ must precede $A_{u}$, while a different module posts the goal of getting the user to achieve the objective by executing $A_{s}$ instead of $A_{u}$. While each of these communicative goals might be well-motivated and coherent in isolation, together they are incoherent, since the first presumes that $A_{u}$ will be executed, while the second recommends retracting the intention to perform $A_{u}$. A text planner with access to both of these top-level communicative goals and their text plans can recognize this implicit conflict and revise and integrate the text plans to resolve it.

There are many ways to unambiguously resolve this class of implicit conflict. Strategy selection depends on the social relationship between the system and the user, as captured by three of RTPIs parameter settings. This relationship is defined by the relative levels of knowledge, expertise, and responsibility of the system and user. Three strategies used by our system, and their motivations, are:

I. Discard communicative goals that implicitly conflict with a system recommendation. In the above example, this would result in a text plan that only recommends doing $A_{s}$ 


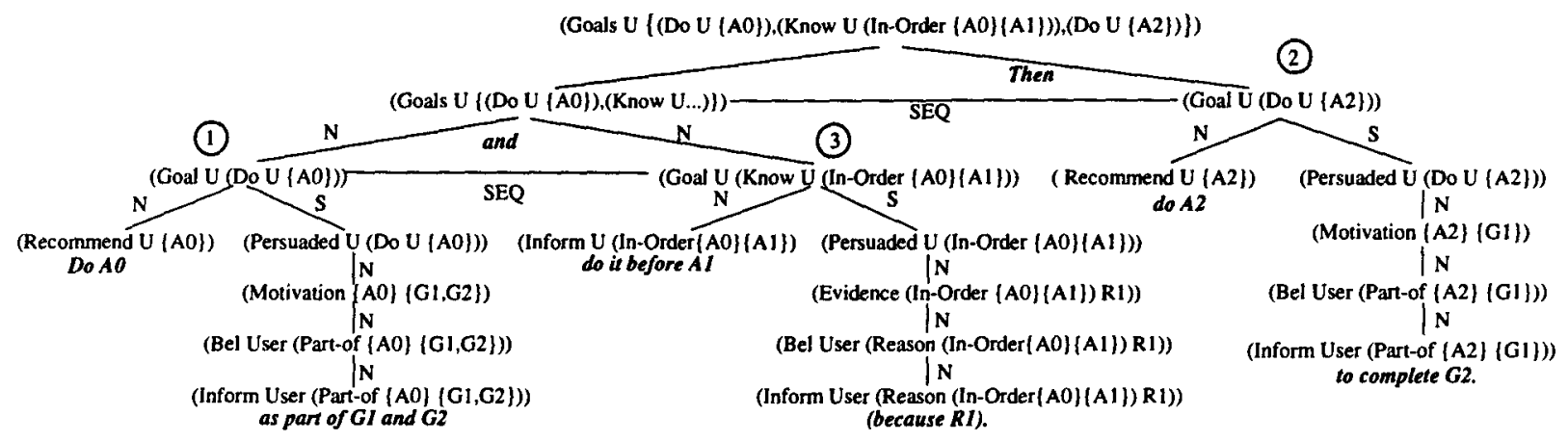

Figure 4: Result of two rules applied to input shown in Fig. 5. First, a rule that applies to trees with top level goals of the form (GOAL USER (DO ...)) uses two trees from Fig. 5 to make a tree with the two subtrees labelled (1) and (2). Next, a rule that places scheduling trees ( (GOAL U (KNOW U (IN-ORDER ...))) ) with related goals inserts a third subtree (3), in this case the entire scheduling tree. A domain specific realizer traverses the tree and inserts cue words and conjunctions based on relations.

instead of $A_{u}$. This strategy would be appropriate if the system is an expert in the domain, has full knowledge of the current situation, and is the sole arbiter of correct performance.

II. Integrate the text plan that implicitly conflicts with the system recommendation as a concession that the user may choose not to accept the recommendation. This strategy is appropriate if the system is an expert in the domain, but the user has better knowledge of the current situation and/or retains responsibility for selecting the best plan of action. Decision support is such an environment. The top half of Figure 6 presents two TraumaTIQ critiques that exhibit implicit conflict, while the bottom part presents the English realization of the integrated text plan, which uses a CONCESSION relation to achieve coherence.

III. Present the system recommendation as an alternative to the user plan. This may be appropriate if the parameters indicate the user has more complete knowledge and more expertise.

\subsubsection{Exploiting Related Goals}

Occasionally two text plans may exhibit no conflict, yet the relationships between their communicative goals can be exploited to produce more coherent text. For example, consider the following two individual critiques produced by TraumaTIQ:

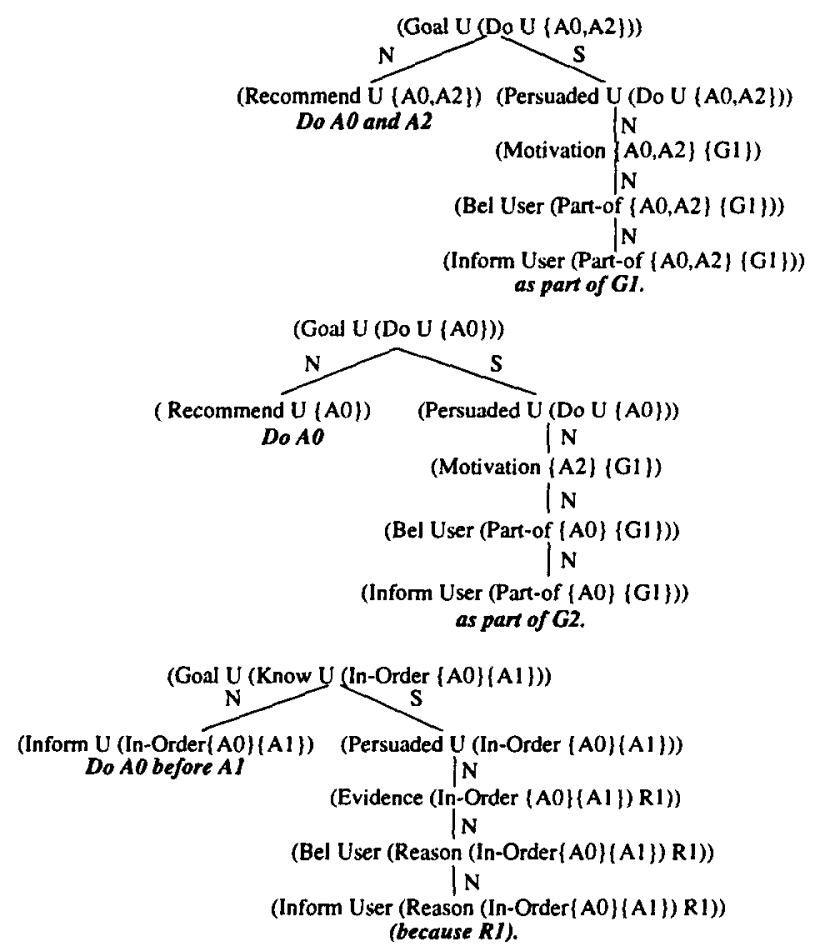

Figure 5: Input to RTPI (see Figure 4).

Caution: do a peritoneal lavage immediately as part of ruling out abdominal bleeding.

Do not reassess the patient in 6 to 24 hours until after doing a peritoneal lavage. The outcome of the latter may affect the need to do the former. 
While the two critiques do not conflict, RTPIs rules exploit the relation between the communicative goals in their respective text plans to produce a more concise and coherent message. In particular, one of RTPIs rules recognizes the interaction between an initial plan to get the user to perform an action $A_{s}$, and a second plan that gets the user to recognize a dependency between $A_{s}$ and another action. This rule creates a text plan for the message:

Do a peritoneal lavage immediately as part of ruling out abdominal bleeding. Use the results of the peritoneal lavage to decide whether to reassess the patient in 6 to 24 hours.

\subsection{Trailing Comments}

Occasionally when several text plans are integrated into a single text plan, another text plan that overlaps with the integrated plan will remain outside the new plan because the scoring function for the applicable rule was too low to allow it to combine. This is typically because an effort to integrate such a text plan would create a message so complex that the heuristic deemed it inappropriate.

However, once concepts have been introduced in the integrated text plan, focusing heuristics (McKeown, 1985) suggest that other text plans containing these concepts be included in the integrated plan as well. Rather than restructure the result of our transformation (against the advice of our heuristic), we append them to the end of the message. Thus we refer to them as trailing comments.

Unfortunately, when the communicative goal is to get the user to perform an action, trailing comments that refer to such actions have the potential to erroneously suggest new instances of actions. Our solution to this problem is implemented in the text realization templates, where we (1) make the focused action the subject of the sentence, reflecting its given status in the discourse, (2) utilize clue words to call attention to its occurrence earlier in the message and to the new information being conveyed, and (3) subordinate other concepts presented with the focused concept by placing them in a phrase introduced by the cue words "along with". In one such example from the trauma domain, the main text plan contains the communicative goal of getting the user to perform several actions, including a laparotomy. A SEQUENCE relation is used to adjoin an overlapping text plan as a trailing comment, and this additional communicative goal is realized in English as (clue words underlined):
TraumaTIQ critiques:

Performing local visual exploration of all abdominal wounds is preferred over doing a peritoneal lavage for ruling out a suspicious abdominal wall injury.

Please remember to check for laparotomy scars before you do a peritoneal lavage.

Message from RTPI integrated plan:

Performing local visual exploration of all abdominal wounds is preferred over doing a peritoneal lavage for ruling out a suspicious abdominal wall injury. However, if you do a peritoneal lavage, then remember to first check for laparotomy scars.

Figure 6: Conflict resolution.

Moreover, doing the laparotomy is also indicated, along with repairing the left diaphragm, to treat the lacerated left diaphragm.

\section{Algorithm}

$R T P I$ performs rule-based integration of a set of RST-style trees. Rules are applied in an order designed to maximize derived benefit. The system first applies the rules that resolve conflict, since we hypothesize that the presence of conflict will most seriously hamper assimilation of a message. Next, the rules that exploit relations between text plans are tried because they enhance coherence by explicitly connecting different communicative goals. Then the aggregation rules are applied to improve conciseness. Finally, the rules for trailing comments reduce the number of disconnected message units.

The algorithm is both greedy and anytime (Garvey and Lesser, 1994); it takes the best result from a single application of a rule to a set of text plans, and then attempts to further apply rules to the modified set. The rule instantiation with the highest heuristic score is chosen and the rule's operator is applied to the trees using those bindings. Since the rules are designed to apply incrementally to a set, every application of a rule results in an improvement in the conciseness or coherence of the tree set, and the tree set is always a viable set of text plans. The user can thus set a time limit for processing of a tree set, and the algorithm can return an improved set at any time. In practice, however, the processing has never taken more than 1-2 seconds, even for large (25 plans) input sets. 


\section{Results}

We tested RTPI using the corpus of critiques generated by TraumaTIQ. A set of critiques was extracted from the middle of each of 48 trauma cases, and RST-style text plans were automatically generated for all the critiques. Then RTPI ran each set, and messages resulting from a template-based realization of RTPIs text plans were analyzed for conciseness and coherence. We are currently using templates for sentence realization since we have been working in the domain of trauma care, where fast real-time response is essential.

There was a $18 \%$ reduction in the average number of individual text plans in the 48 sets examined. The results for individual sets ranged from no integration in cases where all of the text plans were independent of one another, to a $60 \%$ reduction in sets that were heavily inter-related. More concise messages also resulted from a $12 \%$ reduction in the number of references to the diagnostic and therapeutic actions and objectives that are the subject of this domain. The new text plans also allowed some references to be replaced by pronouns during realization, making the messages shorter and more natural.

To evaluate coherence, messages from twelve cases $^{1}$ were presented, in randomly ordered blind pairs, to three human subjects not affiliated with our project. The written instructions given to the subjects instructed them to note whether one set of messages was more comprehensible, and if so, to note why. Two subjects preferred the new messages in 11 of 12 cases, and one subject preferred them in all cases. All subjects strongly preferred the messages produced from the integrated text plan $69 \%$ of the time.

\section{Summary}

Integration of multiple text plans is a task that will become increasingly necessary as independent modules of sophisticated systems are required to communicate with a user. This paper has presented our rule-based system, RTPI, for accomplishing this task. RTPI aggregates communicative goals to achieve more succinct text plans, resolves conflict among text plans, and exploits the relations between communicative goals to enhance coherence.

$R T P I$ successfully integrated multiple text plans to improve conciseness and coherence in the trauma care domain. We will further explore the application of RTPIs domainindependent rules by applying the system to a

\footnotetext{
${ }^{1}$ The evaluation examples consisted of the first eleven instances from the test set where RTPI produced new text plans, plus the first example of conflict in the test set.
}

different domain. We would also like to develop more domain-independent and some domaindependent rules, and compare the fundamental characteristics of each.

\section{References}

Douglas E. Appelt. 1985. Planning english referring expressions. Artificial Intelligence, 26(1):1-33.

Charles B. Callaway and James C. Lester. 1997. Dynamically improving explanations: A revision-based approach to explanation generation. In Proceedings of the 15th International Joint Conference on Artificial Intelligence, Nagoya, Japan, August. IJCAI.

Alan Garvey and Victor Lesser. 1994. A survey of research in deliberative real-time artificial intelligence. The Journal of Real-Time Systems, 6.

A. Gertner and B. L. Webber. 1996. A Bias Towards Relevance: Recognizing Plans Where Goal Minimization Fails. In Proceedings of the Thirteenth National Conference on Artificial Intelligence, Portland, OR.

Eduard Hovy. 1991. Approaches to the planning of coherent text. In Natural Language Generation in Artificial Intelligence and Computational Linguistics, pages 153198. Kluwer.

William C. Mann and Sandra A. Thompson. 1987. Rhetorical structure theory: A theory of text organization. Technical Report ISI/RS-87-190, ISI/USC, June.

Kathleen R. McKeown. 1985. Text Generation. Cambridge University Press, Cambridge, New York.

Johanna Moore and Cecile Paris. 1993. Planning text for advisory dialogues: Capturing intentional and rhetorical information. Computational Linguistics, 19(4):651-695.

Johanna D. Moore, 1995. Participating in Explanatory Dialogues, chapter 3. MIT Press.

Leo Wanner and Eduard Hovy. 1996. The HealthDoc sentence planner. In Proceedings of the International Workshop on Natural Language Generation, pages 1-10.

Bonnie L. Webber, Ron Rymon, and John R. Clarke. 1992. Flexible support for trauma management through goal-directed reasoning and planning. Artificial Intelligence in Medicine, 4:145-163.

Ingrid Zukerman and Richard McConachy. 1995. Generating discourse across several user models: Maximizing belief while avoiding boredom and overload. In Proceedings of the International Joint Conference on Artificial Intelligence, pages 1251-1257. 